\title{
Information Measures over Intuitionistic Four Valued Fuzzy Preferences
}

\author{
Camilo Franco, J. Tinguaro Rodríguez and Javier Montero \\ Faculty of Mathematics, Complutense University, Madrid 28040, Spain
}

\begin{abstract}
This paper studies the meaning of fuzzy preferences under the light of intuitionistic fuzzy sets. Intuitionism is revisited and intuitionistic fuzzy preference relations are defined. A linguistic interpretation of strict and weak fuzzy preferences is then developed where indecision can be explicitly analyzed with the help of a bilattice. Uncertainty is finally examined as a measure of ignorance associated to the intensity value of the preference predicate, where some information measures are applied over its complete structure.
\end{abstract}

\section{INTRODUCTION}

$\mathrm{I}_{\mathrm{s}}$ 's important to search for qualitative and symbolic preference representations so different and complementary methods can exist as a real alternative over the classical body of knowledge found in utility [24] and value theory [5]. In particular, the expressive power of any preference structure is of outmost importance, so a sufficient and complete understanding of the rational decision problem can be achieved. Here we will work on an alternative intuitionistic and linguistic interpretation of the commonly used preference structure found in [9] and [21].

Now, considering diverse studies such as [7] and [10], which follow the ideas presented in [17] and [18], some arguments are found supporting the hypothesis that preferences do not depend solely on the degree of uncertainty over the quality of information, but also over its source, which results in an uncertain outcome due to ignorance. This evidence rise against the descriptive validity of expected utility theory, which motivates us to examine some information measures [11] in order to construct a degree of uncertainty that takes into account the decision agent's natural lack of absolute knowledge.

This paper is organized as follows: section II examines some fuzzy and intuitionistic definitions. The third section takes a first look over the reinterpretation of fuzzy preferences as part of language, following [27], [29], [34].

Manuscript received January 31, 2010.

Camilo Franco is the corresponding author.

e-mail: francodelosrios@gmail.com.
The fourth section studies indecision with the help of a bilattice and the last section reflects on the intuitionistic constructive principle [4], [15] over uncertainty, where some information measures are studied.

\section{ATANASSOV'S INTUITIONISTIC FUZZY PREFERENCES}

Zadeh's fuzzy sets were presented in 1965 (see [32], [33]) and Atanassov's intuitionistic fuzzy sets were introduced in 1986 (see [3]). The following two definitons mark the place for the development of the present fuzzy preference analysis. Have in mind that the common valuation space $[0,1]$ is just an example of a more general definition, due to [13], of a partially ordered structure $L$.

Definition 1. [32]: A fuzzy set $A$ in $X=\{x\}$ is given by $A=\left\{\left\langle x, \mu_{A}(x)\right\rangle \mid x \in X\right\}$, where $\mu_{A}(x): X \rightarrow L$ is the membership function of the fuzzy set $A, L$ is a complete valuation space with a partially ordered structure [13] and $\mu_{A}(x) \in L$ is the membership intensity or value for every $x \in X$ in $A$.

Definition 2. [3]: An intuitionistic fuzzy set $I A$ in $X$ is given by $I A=\left\{\left\langle x, \mu_{I A}(x), v_{I A}(x)\right\rangle \mid x \in X\right\}$, where $\mu_{I A}(x): X \rightarrow L$ is the degree of membership and $v_{I A}(x): X \rightarrow L$ is the degree of non-membership of the fuzzy set $I A$ : associated to each intuitionistic set $I A$ in $X$ there is an uncertainty or hesitancy degree about the membership of $x$ to $A$,

$\pi_{I A}(x)=\max [L]-\mu_{I A}(x)-v_{I A}(x)$,

where $\min [L] \leq \pi_{I A}(x) \leq \max [L]$.

Now, in the field of preference-decision theory, the problem of decision making may be understood as the construction of an ordering process [1] over a finite set of alternatives $O$. If the real decision responds to such a process or not is still to be determined until a better understanding between reality, thought and language exists. But first, the dimensions for preference analysis have to be introduced (see for example [14]). These dimensions are 
here understood as the necessary criteria for the construction of a certain viewpoint under which a subset of the alternatives in $O$ can be partially organized (the following definition has been studied in more detail in [11]):

Definition 3. A viewpoint $h \in H$ for a subset of alternatives $S_{h} \subseteq O$ is characterized by an outcome space $\Omega_{h}$, a set of criteria $C_{h}$ where each criterion $c_{h}: S_{h} \rightarrow \Omega_{h}$ maps alternatives to their outcomes, and a partial order given by the set of fuzzy preference relations $R$ over $\Omega_{h}$.

The set of criteria $C_{h}$ is a finite set whose elements can be combined for the construction of any viewpoint $h \in H$, where every criterion determines a new dimension for the outcome space $\Omega_{h}$. For more details on this approach, where a more general definition of a viewpoint can be found, the interested reader may go to [16].

Fuzzy preference relations can now be defined as a partial order over the referential set of available alternatives:

Definition 4. A fuzzy preference relation for a set of available alternatives $O$ is characterized by $F R^{h}=\left\{\left\langle a, \mu_{F R}^{h}(a)\right\rangle \mid a \in O\right\}$, a complete valuation space $L$ where $\mu_{F R}^{h}(a): O \rightarrow L$ is the membership function of the fuzzy set $F R^{h}$ under viewpoint $h$, and $\mu_{F R}^{h}(a) \in L$ is the membership intensity or value for every $a \in O$ in $F R^{h}$, according to the predicate " $a$ is at least as good as any other available alternative under viewpoint $h$ ".

Notice that the aggregated fuzzy preference relation defined above is understood here as a partially ordered set $F R^{h}(L, \leq)$. Such poset can be constructed from smaller pieces of information in a pairwise manner, more precisely, from different binary fuzzy preference relations defined over the same set of alternatives.

Definition 5. A binary fuzzy preference relation for a set of available alternatives $O$ is characterized by the fuzzy set $R^{h}=\left\{\left\langle(a, b), \mu_{R}^{h}(a, b)\right\rangle \mid a, b \in O\right\}$ under a complete valuation space $L$ such that $\mu_{R}^{h}(a, b): O \times O \rightarrow L$ is the membership function of the fuzzy set $R^{h}$ under viewpoint $h$, and $\mu_{R}^{h}(a, b) \in L$ is the membership intensity or value for every $a, b \in O$ in $R^{h}$, according to the predicate " $a$ is at least as good as $b$ under viewpoint $h$ ".
In an analogous way as intuitionistic fuzzy sets, an intuitionistic binary fuzzy preference relation can be characterized as follows:

Definition 6. An intuitionistic binary fuzzy preference relation $I R^{h}$ in $O$ is characterized, $\forall a, b \in O$, by

$I R^{h}=\left\{\left\langle(a, b), \mu_{I R}^{h}(a, b), v_{I R}^{h}(a, b), \pi_{I R}^{h}(a, b)\right\rangle\right\}$,

where $\mu_{I R}^{h}(a, b): O \times O \rightarrow L$ is the degree of membership, $v_{I R}^{h}(a, b): O \times O \rightarrow L$ the degree of non-membership and $\pi_{I R}^{h}(a, b)$ is the degree of uncertainty or hesitancy of the fuzzy set $I R^{h}$ under viewpoint $h$, where

$\pi_{I R}^{h}(x)=\max [L]-\mu_{I R}^{h}(x)-v_{I R}^{h}(x)$,

such that $\min [L] \leq \mu_{I R}^{h}(x)+v_{I R}^{h}(x) \leq \max [L]$ and $\min [L] \leq \pi_{I R}^{h}(x) \leq \max [L]$.

Notice that from the constructivist principle of intuitionistic logic, as understood by [4], [15], the degree of uncertainty should not be defined for an intuitionistic fuzzy set by an excluding method as it is done with $\pi_{I A}(x)=1-\mu_{I A}(x)-v_{I A}(x), \quad$ where $L=[0,1] . \quad$ If the construction of $\pi_{I A}(x)$ is questioned and independently determined, then the restriction

$\min [L] \leq \mu_{I R}^{h}(x)+v_{I R}^{h}(x) \leq \max [L]$

would not be necessary.

In this paper the appropiateness of the term intuitionistic [3] will not be questioned, but rather it will be studied as it has been accepted by so many in the fuzzy community. In the following sections it will be presented a proposal for understanding and applying intuitionistic fuzzy sets over preferences, so the indecision or uncertainty intensities can be positively constructed rather than defined by exclusion (on some pertinent reflections over this and related issues see [20]).

Now, according to the characterization of the binary valued relation $R$, as a function $R: O \times O \rightarrow L$, the complementation, dual, and inverse of $R$ can now be defined, $\forall a, b \in O$, as [9]:

$R^{c}(a, b)=n(R(a, b))$

$R^{-1}(a, b)=R(b, a)$

$R^{d}(a, b)=n(R(b, a))$

where $n$ is a strong negation. 
Following classical set theory, the three binary relations that compose $R$ can be defined as [9]:

$$
\begin{aligned}
& P=R \cap R^{d} \\
& I=R \cap R^{-1} \\
& J=R^{c} \cap R^{d}
\end{aligned}
$$

This preference structure $(P, I, J)$ should then fullfill the following classical properties:

(1) $P \cup I=R$

(2) $P \cup I \cup P^{-1}=R \cup R^{-1}$

(3) $P \cup J=R^{d}$

(4) $P \cup P^{-1} \cup I \cup J=O \times O$

By (4), and following [9], [21], a binary fuzzy preference relation is a composition of four different fuzzy binary relations, which can be introduced with the following axiom:

Axiom 1. Independence of Irrelevant Alternatives: for every pair of alternatives $a, b \in O$, the values of strict preference $P^{h}(a, b)$, indifference $I^{h}(a, b)$, and incomparability $J^{h}(a, b)$, depend only on the alternatives $a, b$ and the viewpoint $h$ by which they are judged inside the valuation space $L$, where $\mu_{P, I, J}^{h}(a, b): O \times O \rightarrow L$, according to the degree in which " $a$ is better than $b$ under viewpoint $h^{\text {": }}$ $\mu_{P}^{h}(a, b) \in L, " a$ is as good as $b$ under viewpoint $h "$ : $\mu_{I}^{h}(a, b) \in L$, and " $a$ is not anything as $b$ under viewpoint $h^{\prime \prime}: \mu_{J}^{h}(a, b) \in L$.

A fuzzy binary preference relation can now be defined with its complete structure in an alternative way:

Definition 7. A binary fuzzy preference relation $R^{h}$ in $O=\{a, b\}$ is given by:

$$
R^{h}=\left\{\left\langle(a, b), \mu_{P}^{h}(a, b), \mu_{P^{-1}}^{h}(a, b), \mu_{I}^{h}(a, b), \mu_{J}^{h}(a, b)\right\rangle\right\}
$$

where $\mu_{P, I, J}^{h}(a, b): O \times O \rightarrow L$ are the membership functions of the fuzzy set $R^{h}$ under viewpoint $h$, and $\mu_{P, I, J}^{h}(a, b) \in L$ are the membership intensities or values for every $a, b \in O$ in $R^{h}$, according to the de-composition of the predicate " $a$ is at least as good as $b$ under viewpoint $h$ " into the four predicates " $a$ is better than $b$ under viewpoint $h$ ", $\mu_{P}^{h}(a, b) \in L, " b$ is better than $a$ under viewpoint $h "$, $\mu_{P^{-1}}^{h}(a, b) \in L, \quad " a$ is as good as $b$ under viewpoint $h "$, $\mu_{I}^{h}(a, b) \in L$, and " $a$ is not anything as $b$ under viewpoint $h^{\prime \prime}, \mu_{J}^{h}(a, b) \in L$.
It naturally results from definition 7 , in the same way as proposed in [21], that preference modelling is basically an aggregation problem. The first basic evaluation for a decision maker is to find the degree of comparability and incomparability between the alternatives at hand. Such evaluations occur in a first and more basic level (inside a sequential and extensive learning process over the possible viewpoints $h \in H$ ), where comparability intensity is nothing more than the aggregation of strict preference, its opposite, and indifference $\left(P \cup I \cup P^{-1}\right)$. Therefore, incomparability intensity can be identified as the opposite of comparability. This argument will be discussed in the following section.

\section{ON THE PREDICATE OF PREFERENCE: STRICT AND WEAK PREFERENCE}

Up to now, two definitions on fuzzy sets have been presented with the main goal of defining fuzzy preference relations over Zadeh's fuzzy sets and Atanassov's intuitionistic fuzzy sets. What brings our attention now is the preference predicate, which we have freely used along the way but needs some careful examination. Here we propose a linguistic close up following the lines of [27], [34].

Fuzzy logic offers a variety of uses for different (linguistic) connectives such as conjunction, disjunction or negation, focusing on the semantics and the way to use certain syntaxis. Just as stressed in [27], [29], the understanding of language implies to know what its words mean and how to use them properly. In particular, here it's examined the meaning of the strict preference predicate " $a$ is better than $b^{\prime \prime}$ and the weak preference predicate, which results from the aggregation of strict preference and indifference, " $a$ is at least as good as $b "$.

Let's start with the weak preference relation $R$, which stands for the predicate " $a$ is at least as good as $b$ ", expressesing an ordering relation that has to be fully understood through its four valued complete structure: $R=\left\{P, P^{-1}, I, J\right\}$. Just as happens with language, to capture the meaning and use of words in an expression forces us to consider, besides the words alone, the expression as a whole. That's why $R$ can't be only understood without its complete structure, neither can $P$ or $I$ or $J$ can be integrally understood each one by itself, but related with $R$ by some subyacent mapping.

Now, if we analyze (motivated on the general fuzzy interpretation of [27]) the weak preference predicate and its application over the set of alternatives $O$, assigning an order in $O$ according to the meaning of the predicate $R$, we have to verify to what extent does $a$ has the property of being "at least as good as any other alternative", evaluating pair by pair to what extent " $a$ is at least as good as $b$ " for every $a, b \in O$. Therefore, we have to determine if $a$ verifies the property expressed by $R$ and to what extent it does: a poset $\mathrm{Z}=\left(L_{R}^{h}, \leq\right)$, where $\gamma=\inf Z$ and $\lambda=\sup Z$, and a 
membership function $\mu_{R}^{h}: O \rightarrow L_{R}^{h}$ associated to a viewpoint $h$ is assumed to exist from definition 4 , verifying to what extent $a$ is $R$.

This empirical verification is not an easy task. If no positive verification of $R$ is possible or a high degree of uncertainty is associated to it, then some similar relation may be established for evaluating to what extent a questioned pair of alternatives verify certain property. Let's take for example the predicate $P$ : " $a$ is better than $b$ ", whose property is that one of being "better" or "more preferred". It can be accepted that $P$ has a similar meaning as $R$, defining a certain proximity in their intensities, but as it may occur that they can coincide (in the case that $\mu_{R}^{h}(a, b)=\mu_{P}^{h}(a, b)$ ), they can also diverge.

The introduction of a third element would make the evaluation of such a proximity much more robust and informative. If the most distant relation from $P$, or its opposite, is located, then the third element could be its antonym, understanding this term as it's commonly used: the negate of $P$ is its greatest antonym (in the cases where the negate and the antonym differ, the antonym is then a regular one). If the function $\mu_{P}^{h}(a, b)$ expresses to which extent the predicate $P=$ "better than" exists, then the predicate $P^{-}=$"worst than" exists as the antonym of $P$, in such a way that $\mu_{P^{-}}^{h}(a, b)=\mu_{P^{-1}}^{h}(a, b)$.

As stated above and from definition 7 , the predicate $P$ can also be interpreted as "better than", where $P^{-}$would be read as "worse than". The polarity of these linguistic terms allows us to start the construction of the subyacent mapping between $R$ and $P, P^{-}$. Following (1) and (2), the link between a strict preference, its opposite, and weak preference is indifference. Such predicate $I$ expresses the relation "as good as", which can be aggregated with strict preference for knowing up to what extent the property of being "at least as good as" is verified. But in the same way as we have done with the preference predicate, the indifference one has an opposite interpretation: "as bad as".

In order to clarify this last idea, let's analyze first the predicate $J$, "not anything as", of incomparability. If we want to find out to what extent an alternative is uncomparable with another, then we have to evaluate the negate of comparability. Such predicate of comparability expresses the relation "as anything as", which can be deconstructed into two predicates, $I$ and its antonym $I$, "as good as" and "as bad as". Like said before, such evaluations occur in a first and more basic level where a sequential and extensive learning-decision process is taking place. Then, in a second level of analysis, the whole structure for $R$ can be examined, evaluating the existing mapping between $R$ and $P$, $P^{-}, I$, and $I$.

The whole preferential structure for $R$ can now be defined from an intuitionistic perspective, aggregating $P$ and $I$ as the membership degree and $P^{-}$and $I^{-}$as the non-membership degree. The uncertainty degree will be discussed in the following section.

Definition 8. An intuitionistic binary fuzzy preference relation $I R^{h}$ in $O=\{a, b\}$ is characterized by

$I R^{h}=\left\{\left\langle(a, b), \mu_{I R}^{h}(a, b), v_{I R}^{h}(a, b), \pi_{I R}^{h}(a, b)\right\rangle\right\}$,

where $\mu_{I R}^{h}(a, b): O \times O \rightarrow L$ is the degree of membership, defined as $\mu_{P}^{h}(a, b)+\mu_{I}^{h}(a, b), v_{I R}^{h}(a, b): O \times O \rightarrow L$ is the degree of non-membership, defined as $v_{P^{-}}^{h}(a, b)+v_{I^{-}}^{h}(a, b)$, and $\pi_{I R}^{h}(a, b)$ is the degree of uncertainty, defined as

$\pi_{I R}^{h}(a, b)=\lambda-\mu_{I R}^{h}(a, b)-v_{I R}^{h}(a, b)$,

of the fuzzy set $I R^{h}$ under viewpoint $h$, such that, $\forall a, b \in O$, $\gamma \leq \mu_{I R}^{h}(a, b)+v_{I R}^{h}(a, b) \leq \lambda$ and $\gamma \leq \pi_{I R}^{h}(a, b) \leq \lambda$.

Until a constructive proposal for $\pi_{I R}^{h}(x)$ is not obtained, the condition $\gamma \leq \mu_{I R}^{h}(a, b)+v_{I R}^{h}(a, b) \leq \lambda$ will continue as a key property necessary for the definition of the intuitionistic uncertainty degree.

\section{FOUR VALUED INTERPRETATION OF INTUITIONISTIC FUZZY PREFRENCES}

As reasoned in the previous section, the weak preference relation $R$, expressed by the predicate "at least as good as", results from the aggregation of $P$ and $I$, where $P \cup I$ composes the predicate "at least as good as" and $P^{-} \cup I^{-}$ composes the predicate "at least as bad as". The first one expresses the intensity of positive comparability between any pair of alternatives while the second one expresses its opposite intensity, that one of antagonistic comparability. Now the preference structure offers the possibility to value the reasons why an alternative is "at least as good as", and the reasons why an alternative is "at least as bad as" any other alternative.

Notice that in definition 8 , the uncertainty measure is defined without constructing it, but rather as everything that is not membership and non-membership. It has been pointed out that intuitionistic fuzzy sets [4], [15] require a positive mathematical mental construction in order to state the existence of such a mathematical concept. In other words, the uncertainty valuation needs to be constructed in such a way that we can properly examine the boundary between membership and non-membership.

What we need is a representation of the subyacent mapping between the different components of $R$ as we have seen them here, so the uncertainty component can be constructively obtained as a measure over the available information. A natural possibility for the treatment of this 
information could be the four-valued logic (FOUR) of Belnap [2] (for a complementary exposition of the principle of four-cornered negation the interested reader can see [23]). This logic originally presented a way for dealing with inconsistent and incomplete information, on one hand evaluating the truth of a predicate and, on the other hand, the available information, where no information gave account of ignorance and over-information of inconsistency. Generalizing Belnap's logic, M. Ginsberg (1988, in [2]) proposed the use of algebraic structures, called bilattices, where FOUR is the minimal nondegenerated bilattice [2].

FOUR has been used before over preference modelization, such as in $D D T$ logic [22], [28], where four values are used, following Belnap's logic: truth $(t)$, falsehood $(f)$, ignorance $(u)$, and inconsistency $(k)$. In this interpretation, the situation $t$ exists when there are positive reasons for accepting proposition $P$ and no negative reasons against it, $f$ exists if there are no positive reasons but only negative reasons against $P, u$ exists when there are no positive nor negative reasons for or against $P$, and $k$ exists when there are positive and negative reasons for and against $P$. The present work proposes a different interpretation based on intuitionistic fuzzy preferences (definition 8) and certain phylosophical matters [23].

According to the ideas developed in the past sections, the comparative analysis between any pair of alternatives under a certain viewpoint starts when the whole structure for $R$ is examined, evaluating the existing mapping between $R$ and $P$, $P^{-}, I$, and $I$. Such components will be now distributed according to a bilattice where an arbitrary number of truth values, based on the decision agent's knowledge, can be arranged into two different orders, each forming a lattice (one preference lattice and another indecision lattice). Their aggregation, in the form of a bilattice, will then result in a final value for $R$.

The mapping of the complete comparative structure of $R$ is pictured in figure 1. The bilattice assings on one dimension the truth of $P$ and $P^{-}$and on the other the truth of $I$ and $I^{-}$. In particular, when $L=[0,1]$, the order that the preference lattice assigns on the alternatives is non-reflexive: $\mu_{P}^{h}(a, a) \geq 0$ and $v_{P^{-}}^{h}(a, a) \geq 0$, transitive: if $\mu_{P}^{h}(a, b)>0$ and $\mu_{P}^{h}(b, c)>0$ then $\mu_{P}^{h}(a, c)>0$ or if $v_{P^{-}}^{h}(a, b)>0$ and $v_{P^{-}}^{h}(b, c)>0$ then $v_{P^{-}}^{h}(a, c)>0$, and anti-symmetric in the following sense: if $\mu_{P}^{h}(a, b)>0$ and $v_{P^{-}}^{h}(a, b)>0$ then $a$ and $b$ can be identified as substitutes or equivalent alternatives up to a certain degree, where $\mu_{I}^{h}(a, b)>0$ and/or $v_{I^{-}}^{h}(a, b)>0$.

On the other hand, the order that the indecision lattice assigns on the alternatives is reflexive: $\mu_{I}^{h}(a, a) \leq 1$ and $v_{I^{-}}^{h}(a, a) \leq 1, \quad$ anti-symmetric: if $\quad \mu_{I}^{h}(a, b)>0 \quad$ and $v_{I^{-}}^{h}(a, b)>0$ then $a$ and $b$ are up to a certain degree equivalent alternatives (if $\mu_{I}^{h}(a, b)=v_{I^{-}}^{h}(a, b)>0 \quad$ no difference between them can be found under viewpoint $h$ ), and non-transitive in the following sense: if $\mu_{I}^{h}(a, b)>0$ and $\mu_{I}^{h}(b, c)>0$ then $\mu_{I}^{h}(a, c) \geq 0$ or if $v_{I^{-}}^{h}(a, b)>0$ and $v_{I^{-}}^{h}(b, c)>0$ then $v_{I^{-}}^{h}(a, c) \geq 0$ (the reader can find a detailed discussion on intransitivity of indifference in [19]).

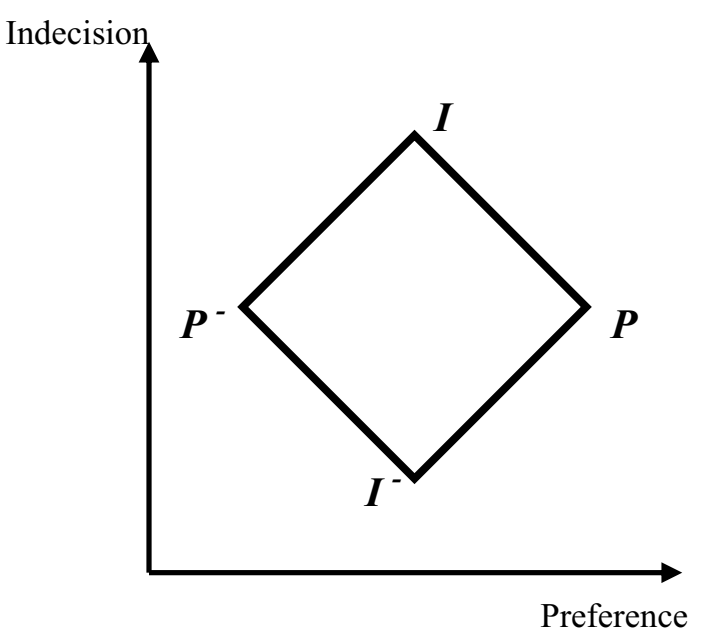

Figure 1. Bilattice over the complete preference structure $R$.

Notice that indecision is a common linguistic synonym of uncertainty, but it's not the kind of uncertainty that intuitionistic fuzzy sets or preference relations intend to formalize. The main goal now is to examine the construction of an uncertainty degree over the valuation procedure in a fuzzy preference relation. In particular, the kind of uncertainty that we focus on is the one that exists due to ignorance, mainly as lack of knowledge, a natural state of mind for any intelligent and rational agent.

\section{INFORMATION MEASURES AND INTUITIONISTIC FUZZY PREFERENCES}

Under the intuitionistic fuzzy approach [3] followed here, we now explore the construction of an uncertainty degree over the preference valuation structure. In a rational decision process, an intelligent agent depends on his/her state of knowledge, in such a way that uncertainty appears to be related to some degree of ignorance on the validity of information, for example, when the source of information is not completely reliable [7], [10]. It can be argued that such uncertainty cannot be measured by probability functions (the original argument can be found in [17], [18], but see [6] for a recent overview on the historical and scientific development of uncertainty representation) and as significant as it is in the construction of a preference order, it should be included as an explicit numerical function in the preference structure. 
Fishburn [8] suggested such function (a qualitative counterpart has also been proposed [30]), called ambiguity, and Yager [31] explored it under a fuzzy environment. We now recall the definition of an ambiguity function: Let $P^{f}(R)$ represent the set of all the fuzzy subsets $R$, such that $\alpha: P^{f}(R) \rightarrow[0,1]$ is an ambiguity measure if and only if it satisfies the following three axioms:

A1. $\alpha(\varnothing)=0$

A2. $\alpha(R)=\alpha\left(R^{\mathrm{c}}\right)$

A3. $\alpha(R \cup B)+\alpha(R \cap B) \leq \alpha(R)+\alpha(B)$

where $R \cup B(x)=\max (R(x), B(x)), R \cap B(x)=\min (R(x), B(x))$ and $R^{\mathrm{c}}(x)=1-R(x)$.

The first axiom assigns minimum ambiguity to the null set. In the second axiom, the complement of $R$ is denoted by $R^{\mathrm{c}}$, so ambiguity exists as a measure of a certain attribute of information shared by any fuzzy set $R$ and its complementation. From A1 and A2 it can be seen that $\alpha(\varnothing)=\alpha(X)=0$. A2 reflects the original intuition [8] that whatever underlies the ambiguity of a set also underlies the ambiguity of its complement. It can be seen the foundational role that complementation (strong negation) has on the construction of $\alpha$. Finally, A3 expresses submodularity: the union of two fuzzy sets $R, B$ may reduce or cancel ambiguities associated to each one (considered separately), in such a way that if $R$ and $B$ are disjoint, then $\alpha(R \cup B) \leq \alpha(R)+\alpha(B) \quad$ and $\quad$ if $\quad \alpha(R \cup B)=X \quad$ then $\alpha(R \cap B) \leq \alpha(R)+\alpha(B)$.

Uncertainty due to ignorance can now be understood regarding the ambiguity function $\alpha$. Notice that membership and non-membership degrees are independent values, where $\mu_{I R}^{h}(a, b)$ and $v_{I R}^{h}(a, b)$ obey to different arguments not necessarily complementary with each other. If we take for example the following ambiguity measure (it's easy to check that it satisfies conditions A1-A3),

$$
\alpha(A)=\frac{1}{n} \sum_{i=1}^{n}\left(A\left(x_{i}\right) \sin A^{c}\left(x_{i}\right)+A^{c}\left(x_{i}\right) \sin A\left(x_{i}\right)\right),
$$

where $A$ is any fuzzy set, $A^{c}$ is the complement of $A$ and $n$ is the cardinality of a set of elements $X$, it could be now possible to assign the ambiguity measure, as an uncertainty degree due to ignorance, to the membership and nonmembership degrees of intuitionistic fuzzy preferences (recall definition 8). In this way, two uncertainty measures will be explicitly assigned to the two independent degrees of membership, $\mu_{I R}^{h}$, and non-membership, $v_{I R}^{h}$.

The membership and non-membership degrees will now have, each one, a corresponding uncertainty degree as an independent measure of ambiguity over its components, where $\mu_{I R}^{h}=\mu_{P}^{h}+\mu_{I}^{h}$ and $v_{I R}^{h}=v_{P^{-}}^{h}+v_{I^{-}}^{h}$. If we consider the following preference intensities,

$$
\begin{aligned}
& \mu_{I R}^{h}(a, b)=\{0.7,0.3\} \text { and } v_{I R}^{h}(a, b)=\{0.2,0.1\}, \\
& \mu_{I R}^{h}(b, c)=\{0.3,0.5\} \text { and } v_{I R}^{h}(b, c)=\{0.6,0.3\}
\end{aligned}
$$

where for any $a, b \in O, \mu_{I R}^{h}(a, b)=\left\{\mu_{P}^{h}(a, b), \mu_{I}^{h}(a, b)\right\}$ and $v_{I R}^{h}(a, b)=\left\{v_{P^{-}}^{h}(a, b), v_{I^{-}}^{h}(a, b)\right\}$, then the respective uncertainty degrees, due to ignorance or lack of knowledge on the membership and non-membership values, are,

$$
\begin{aligned}
& \alpha\left(\mu_{I R}^{h}(a, b)\right)=0.50 \text { and } \alpha\left(v_{I R}^{h}(a, b)\right)=0.31, \\
& \alpha\left(\mu_{I R}^{h}(b, c)\right)=0.54 \text { and } \alpha\left(v_{I R}^{h}(b, c)\right)=0.55 .
\end{aligned}
$$

Notice that the ambiguity value is of a different nature from that one of an intensity value. This is a pertinent warning over the different epistemic states of the numerical values considered here and they are not to be confused. The intensity value, $\mu_{I R}^{h}$ or $v_{I R}^{h}$, is the degree in which the elements of the reference set (in this case alternatives $\forall a, b \in O)$ verify the properties of the predicate involved. On the other hand, ambiguity, $\alpha$, measures the lack of knowledge or uncertainty due to ignorance associated to the precise numerical assignation of the intensity value, but it's not a truth value like the ones of the membership or nonmembership degrees.

As a result, the intuitionistic preference structure given in definition 8 can now be characterized as,

$$
I R^{h}=\left\{\left\langle\mu_{I R}^{h}, v_{I R}^{h}, \alpha\left(\mu_{I R}^{h}\right), \alpha\left(v_{I R}^{h}\right)\right\rangle\right\},
$$

where the uncertainty degree $\pi_{I R}^{h}$ can be decomposed into two values, $\alpha\left(\mu_{I R}^{h}\right)$ and $\alpha\left(v_{I R}^{h}\right)$, and the restrictive condition $\gamma \leq \mu_{I R}^{h}+v_{I R}^{h} \leq \lambda$ can finally be abandoned.

Now, if we want to understand ambiguity as a common link between intuitionistic fuzzy binary preferences, first we have to define the intuitionistic interpretation of the (global) fuzzy preference relation (recall definition 4):

Definition 9. An intuitionistic fuzzy preference relation for a set of available alternatives $O$ is characterized by $I F R^{h}=\left\{\left\langle a, \mu_{I F R}^{h}(a), v_{I F R}^{h}(a), \alpha\left(\mu_{I F R}^{h}\right), \alpha\left(v_{I F R}^{h}\right)\right\rangle \mid a \in O\right\}$,

a complete valuation space $L$ where $\mu_{I F R}^{h}(a): O \rightarrow L$ and $v_{I F R}^{h}(a): O \rightarrow L$ are the membership and non-membership functions of the fuzzy set $I F R^{h}$, according to the predicates " $a$ is at least as good as any other available alternative under viewpoint $h$ " and " $a$ is at least as bad as any other available alternative under viewpoint $h$ ", respectively. The membership $\mu_{I F R}^{h}(a)$ and non-membership degrees $v_{I F R}^{h}(a)$ 
have each one an associated uncertainty degree, $\alpha\left(\mu_{I F R}^{h}\right)$ and $\alpha\left(v_{I F R}^{h}\right)$, defined as an ambiguity measure in the sense of A1-A3.

Let's take for example the following intuitionistic fuzzy preferences, where an alternative $a$ is compared with three other alternatives given the structure $I R=\left\{\mu_{I R}^{h}, v_{I R}^{h}\right\}$ :

$$
\begin{aligned}
& \operatorname{IR}(a, b)=\{0.3,0.1\}, \\
& \operatorname{IR}(a, c)=\{0.7,0.3\}, \\
& \operatorname{IR}(a, d)=\{0.9,0.8\} .
\end{aligned}
$$

In order to identify the decision agent's uncertainty degree, we consider the ambiguity measure $\alpha$ over its positive and negative truth intensities. As a result, following the notation $\alpha(I F R)=\left\{\alpha\left(\mu_{I F R}^{h}\right), \alpha\left(v_{I F R}^{h}\right)\right\}$, we have:

$$
\alpha(\operatorname{IFR}(a))=\{0.32,0.29\}
$$

where the alternative $a$ is weakly better than the rest with greater uncertainty due to ignorance than when it is valued as weakly worst. It would then be necessary to compare the values of IFR for the other alternatives and decide over the available information.

If we consider, for example, the following preference values of the alternative $b$,

$$
\begin{aligned}
& \operatorname{IR}(b, a)=\{0.2,0.1\}, \\
& \operatorname{IR}(b, c)=\{0.8,0.2\}, \\
& \operatorname{IR}(b, d)=\{0.9,0.4\},
\end{aligned}
$$

the corresponding uncertainty degree of $\operatorname{IFR}(b)$ is:

$$
\alpha(\operatorname{IFR}(b))=\{0.26,0.31\} \text {. }
$$

Therefore, weak preference of $b$ over the rest of alternatives obtains a lower degree of uncertainty due to ignorance than the weak preference value of $a$, and a higher degree of uncertainty when valued as weakly worst. In this way, ambiguity offers relevant information and serves as a common link between binary fuzzy preferences, where a rational agent may be able to systematically identify, given a particular decision criterion, the preferred and optimal alternative(s).

\section{CONCLUSION}

Once intuitionistic fuzzy preferences are defined and interpreted, the decision maker has the opportunity to differentiate incomparability from positive and negative strict preference and indecision. The possibility of expressing membership and non-membership intenstities is of the outmost importance, where antagonistic values open expressive possibilities for a better understanding of the decision problem and the identification of second order characteristics of the alternatives at play.

Different information measures can be useful along the learning process of the rational agent, aiding his/her decision under uncertainty. Here we focus on the type of uncertainty due to ignorance, measuring the agent's lack of knowledge as a measure of ambiguity. As a result, a complete preference structure is proposed under the intuitionistic constructive principle and following the lines of Attanassov's intuitionistic fuzzy sets. It is then possible for a rational agent to reveal preference, non-preference, indecision, and the uncertainty due to ignorance associated to such preference values.

\section{REFERENCES}

[1] Amo, A., Montero, J., Biging, G., Cutello, V.: Fuzzy classification systems. European Journal of Operational Research 156, 459-507 (2004)

[2] Arieli, O., Avron, A.: The value of the four values. Artificial Intelligence 102, 97-141 (1997)

[3] Atanassov, K.: Intuitionistic Fuzzy Sets. Theory and Applications. Physica-Verlag, New York (1999)

[4] Brouwer L. Collected Works. North-Holland, Amsterdam (1975)

[5] Debreu, G. Theory of value. Yale University Press, New York (1959)

[6] Dubois, D., Prade, H.: Formal representations of uncertainty. In D. Bouyssou, D. Dubois, M. Pirlot, H. Prade, eds, Decision-Making Process, ISTE, London (2009)

[7] Ellsberg, D.: Risk ambiguity and the savage axioms. Quarterly Journal of Economics 75, 643-669 (1961)

[8] Fishburn, P.: The axioms and algebra of ambiguity. Theory and Decision 34, 119-137 (1993)

[9] Fodor, J., Roubens, M.: Fuzzy Preference Modelling and Multicriteria Decision Support. Kluwer Academic Publishers, Dordrecht (1994)

[10] Fox, C., Tversky, A.: Ambiguity aversion and comparative ignorance. The Quarterly Journal of Economics 110, 585-603 (1995)

[11] Franco de los Ríos, C., Montero, J., Rodríguez, J.: Rectification of fuzzy preferences in a fuzzy environment. IPMU'10 (2010)

[12] García-Lapresta J.L., Montero J.: Consistency of crisp and fuzzy preference relations. Proceedings of the IPMU'04 Conference, pp. 527-534 (2004)

[13] Goguen, J.: L-fuzzy sets. Journal of Mathematical Analysis and Applications 18, 145-174 (1967)

[14] González-Pachón, J., Gómez, D., Montero, J., Yáñez, J.: Soft dimension theory. Fuzzy Sets and Systems 137, 137-149 (2003)

[15] Heyting, A.: Intuitionism, an Introduction. North-Holland, Amsterdam (1966)

[16] Junker, U.: Preferences in an open world. In: First International Conference, ADT 2009, pp. 215-224 (2009)

[17] Keynes, J.: A Treatise on Probability. MacMillan, London (1963)

[18] Knight, F.: Risk, Uncertainty, and Profit. University of Chicago Press, Chicago (1971)

[19] Monguin, Ph.: Does optimization imply rationality? Synthese 124, 73 $111(2000)$

[20] Montero, J., Gómez, D., Bustince, H.: On the relevance of some families of fuzzy sets. Fuzzy Sets and Systems 158, 2429-2442 (2007)

[21] Montero, J., Tejada, J., Cutello, C.: A general model for deriving preference structures from data. European Journal of Operational Research 98, 98-110 (1997)

[22] Öztürk, M., Tsoukiàs, A.: Modelling uncertain positive and negative reasons in decision aiding. Decision Support Systems 43, 1512-1526 (2007)

[23] Raju, P.: The principle of the four-cornered negation in indian philosophy. Review of Metaphysics 7, 694-713 (1954)

[24] Samuelson, P.: A note on the pure theory of consumer's behaviour. Economica, New Series 5, 61-71 (1938) 
[25] Sancho-Royo, A., Verdegay, J.: Coherence measures on finite fuzzy sets. International Journal of Uncertainty, Fuzziness and Knowledgebased Systems 8, 641-663 (2000)

[26] Sancho-Royo, A., Verdegay, J.: Fuzzy coherence measures. International Journal of Intelligent Systems 20, 1-11 (2005)

[27] Trillas, E.: Views of Fuzzy Sets and Systems from Different Perspectives. (Ed.: Rudolf Seising) Springer, pp.175-205 (2009)

[28] Tsoukiás, A.: A first-order, four valud, weakly paraconsistent logic and its relation to rough sets semantics. Foundations of Computing and Decision Sciences 12, 85-112 (2002)

[29] Wittgenstein, L.: Investigaciones Filosóficas. Universidad Autónoma de México, México (2003)

[30] Wong, M., Wang, Z., Bollmann, P.: On qualitative measures of ignorance. International Journal of Intelligent Systems 11, 27-47 (1998)

[31] Yager, R.: On a measure of ambiguity. International Journal of Intelligent Systems 10, 1001-1019 (1995)

[32] Zadeh, L.: Fuzzy sets. Information and Control 8, 338-353 (1965)

[33] Zadeh, L.: The concept of a linguistic variable and its application to aproximate reasoning-I. Information Sciences 8, 199-249 (1975)

[34] Zadeh, L.: Fuzzy logic = computing with words. IEEE Transactions on Fuzzy Systems 4, 103-111 (1996) 